Article

\title{
Synthesis of Star Poly( $N$-vinylcarbazole) by Microwave-Assisted Reversible Addition-Fragmentation Chain Transfer Polymerization (RAFT)
}

\section{Mircea Grigoras * and Oana-Iuliana Negru}

"P. Poni" Institute of Macromolecular Chemistry, Electroactive Polymers Department, 41A Gr. Ghica Voda alley, Iasi-700487, Romania; E-Mail: irimia.oana@icmpp.ro

* Author to whom correspondence should be addressed; E-Mail: grim@icmpp.ro; Tel.: +40-0232-217454; Fax: +40-232-211299.

Received: 27 February 2012; in revised form: 12 April 2012 / Accepted: 4 May 2012 / Published: 15 May 2012

\begin{abstract}
Controlled radical polymerization of $N$-vinylcarbazole (NVK) via microwave-assisted reversible addition-fragmentation chain transfer (RAFT) polymerization is described. As chain transfer agent, 1,3,5-benzyl tri (diethyldithiocarbamate), was used. The chain transfer agent, containing a 1.3.5-trisubstituted benzene ring as core and three dithiocarbamate functionalities attached through an intermediate for fragmenting covalent bonds, led to poly( $N$-vinylcarbazole) (PVK) with star architecture. Polymerizations were carried out in 1,4-dioxane as solvent, at $70{ }^{\circ} \mathrm{C}$, and studied for different polymerization times and monomer/CTA/initiator ratios. The SEC molecular weight curves exhibit a trimodal distribution, assigned to the linear and star-star coupling polymers, accompanying the real star polymer (as main product).
\end{abstract}

Keywords: $N$-vinylcarbazole; RAFT polymerization; three-functional dithiocarbamate chain transfer agent; microwave-assisted polymerization; star polymers

\section{Introduction}

Carbazole-based polymers are materials of considerable scientific and industrial interest due to their attractive features, such as hole-transporting, high charge-carrier mobility, and electroluminescent properties [1,2]. Among carbazole polymers, a central place is reserved for poly $(N$-vinyl carbazole) 
(PVK) because it is easily synthesized, is soluble in common organic solvents and as the poly ( $N$-vinylcarbazole)-2,4,7-trinitro-9-fluorenone (PVK-TNF) charge transfer complex is one of the best known organic photoconducting materials for application in electrophotography [3]. Several polymerization techniques are available and cationic polymerization using almost all known cationic initiators, or radical initiation with azo- and peroxide compounds are the most employed methods for obtaining linear PVK [1]. All previous studies on the charge transfer complexes (CTC) of PVK and their photoconducting properties were carried out using a linear polymer obtained by classical techniques. However, PVK with well-controlled molecular weight and different topologies (linear, star, bloccopolymer, etc.) has been obtained only by controlled polymerization methods. Consequently, it would be very interesting to study the influence of these structural factors on the charge transfer interactions and optoelectronic properties of PVK.

Controlled/"living" radical polymerization (CRP) techniques have attracted significant attention in the past two decades. These methods allow moderate to good control over the polymer chain growth processes and hence synthesis of telechelic polymers and block copolymers became relatively easy using these techniques. Such polymers can be reactivated for a chain extension reaction with the same or different monomers because they possess active end groups. Several methods have been developed for preparing polymers in a controlled living manner; examples are nitroxide-mediated polymerization (NMP) [4,5], atom-transfer radical polymerization (ATRP) [6-9], and reversible addition-fragmentation chain transfer (RAFT) polymerization [10-13]. The reversible addition-fragmentation chain-transfer process has became a very studied process in recent years because it allows the formation of macromolecular compounds with low polydispersity index and complex structures, such as linear and star homopolymers or block-, graft-, comb and star copolymers, using a large variety of functional monomers and suitable chain transfer agents. The whole process is based on conventional free radical polymerization of monomers in the presence of chain transfer agents (CTA), such as: dithiocarbamates, dithioesters, xanthates and trithiocarbonates. The role of CTA is to control molecular weight and polydispersity by preventing the termination of macroradicals as undesired events.

The application of controlled radical polymerization methods to $N$-vinylcarbazole (NVK) is considered difficult, since the propagating radicals are highly reactive due to their non-conjugated nature and strong electron donating pendant groups [14]. The RAFT polymerization of NVK has been reported in two recent papers [14,15] using xanthate-mediated controlled radical polymerization. Diblock copolymers containing poly( $N$-vinylcarbazole) with polyethylene glycol, poly(vinyl acetate), and poly(vinyl alcohol) have also been synthesized by the RAFT process using low molecular and macromolecular xanthates as CTAs [16].

Microwave-assisted chemical synthesis is an employed technique that leads to enhanced rates, reduced reaction times, increased product yields and enhanced product purities by reducing unwanted side reactions compared to conventional heating methods (e.g., with an oil bath or heating block) in which energy is transferred, often via solvent or by thermal conduction [17]. Success in the field of small molecules microwave-assisted organic synthesis [18] has given rise to growing interest in the use of microwaves as a heating source for polymerizations and post-polymerization modifications. The potential ability to accelerate polymerization reactions while simultaneously reducing the probability of side reactions indicates this method may prove particularly useful for the synthesis of well-defined polymers [19]. 
Herein, we report the controlled synthesis of three-arm poly(NVK) star by RAFT polymerization using a three-functional dithiocarbamate as chain transfer agent, with a single-mode microwave reactor. In general, there are two main methods to obtain star polymers: either by linking a given number of linear chains to a central core ("arm-first" method) or by growing branches from an active core ("core-first" method). Additionally two different approaches can be applied for the synthesis of star polymers by RAFT polymerization using suitable CTA agents, the so-called R-group approach (propagation attached to core) and the Z-group approach (propagation away from core) [20-22]. In this study, we selected the R-group approach using a three-functionalized dithiocarbamate, in which the stabilizing groups are linked to the core via a fragmenting covalent bond.

\section{Experimental Section}

\subsection{Materials}

$\mathrm{N}$-Vinylcarbazole (NVK, 98\%) (Aldrich) was recrystallized twice from methanol. 1,3,5-Tris(bromomethyl) benzene $\left(97 \%, \mathrm{mp}=94-99{ }^{\circ} \mathrm{C}\right)$ and sodium $N, N$-diethyldithiocarbamate trihydrate (Aldrich) were used as received. 2,2'-Azobis (isobutyronitrile) (AIBN) was purified by recrystallization from methanol. 1,4-Dioxane was distilled from sodium wire/benzophenone just before use. All other materials were used without further purification.

Synthesis of the trifunctional chain transfer agent: 1,3,5-Benzyl tri(diethyldithiocarbamate) was prepared in a single step route by the reaction of 1,3,5-tribromomethyl benzene with sodium diethyldithiocarbamate (Scheme 1).

Scheme1. Synthesis of the trifunctionalized chain transfer agent.

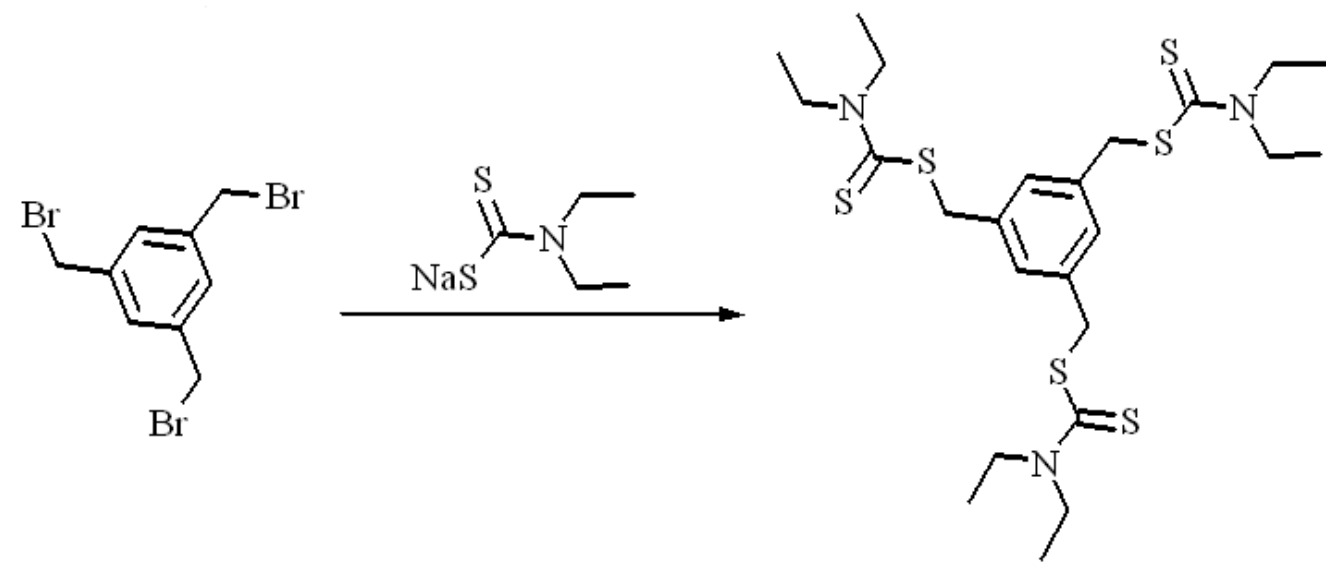

In a $100 \mathrm{~mL}$ two-neck round-bottom flask equipped with magnetic stirrer, inlet-outlet nitrogen and dropping funnel, sodium $N, N$-diethyldithiocarbamate trihydrate ( 2 g $8.87 \mathrm{mmol}$ ) was dissolved in ethanol $(40 \mathrm{~mL})$ under an argon atmosphere and cooled to $0{ }^{\circ} \mathrm{C}$. A solution of 1,3,5-tribromomethyl benzene $(1 \mathrm{~g}, 2.8 \mathrm{mmol})$ in ethanol $(10 \mathrm{~mL})$ was added dropwise over a 30 min period. The reaction was gradually warmed to room temperature, and the mixture was stirred for a further $65 \mathrm{~h}$. The precipitate $(\mathrm{NaBr})$ was filtered off and the product, 1,3,5-benzyl tri (diethyldithiocarbamate), was obtained after evaporating the solvent. The product was recrystallized from ethanol as white plates. 
Yield $=82.3 \% . \mathrm{Mp}=81-82{ }^{\circ} \mathrm{C}$. ESI-MS $(\mathrm{m} / \mathrm{z}): 562.9\left(\mathrm{M}+\mathrm{H}^{+}\right)$. Sulphur analysis: Calcd. For $\mathrm{C}_{24} \mathrm{H}_{39} \mathrm{~S}_{6} \mathrm{~N}_{3}$ (561.8): S; 34.17\%, Found; 33.93\%.

FT-IR (KBr, cm $\left.{ }^{-1}\right)$ : $2931(\mathrm{C}-\mathrm{H})$; 1598, 1488, $1416(\mathrm{C}=\mathrm{C}$ aromatic); $1355(\mathrm{~N}-\mathrm{C}) ; 1068(\mathrm{C}=\mathrm{S})$; 710-916 (C-H from substituted benzene) and $596(\mathrm{C}-\mathrm{S})$. UV $\left(\mathrm{CHCl}_{3}\right): 254$ and $280 \mathrm{~nm} .{ }^{13} \mathrm{C}-\mathrm{NMR}$ $\left(\mathrm{CDCl}_{3}, 100 \mathrm{MHz}\right)$ ppm: 12, $13\left(\mathrm{C}_{\mathrm{a}}\right)$; 46, $50\left(\mathrm{C}_{\mathrm{b}}\right) ; 42\left(\mathrm{C}_{\mathrm{c}}\right) ; 130\left(\mathrm{C}_{\mathrm{d}}\right) ; 157\left(\mathrm{C}_{\mathrm{e}}\right) ; 196\left(\mathrm{C}_{\mathrm{f}}\right)$.

The ${ }^{1} \mathrm{H}$ - NMR spectrum (Figure 1) confirms the structure of the CTA.

Figure 1. ${ }^{1} \mathrm{H}-\mathrm{NMR}$ spectra of three-arm chain transfer agent (CTA) $\left(400 \mathrm{MHz}, \mathrm{CDCl}_{3}\right)$.

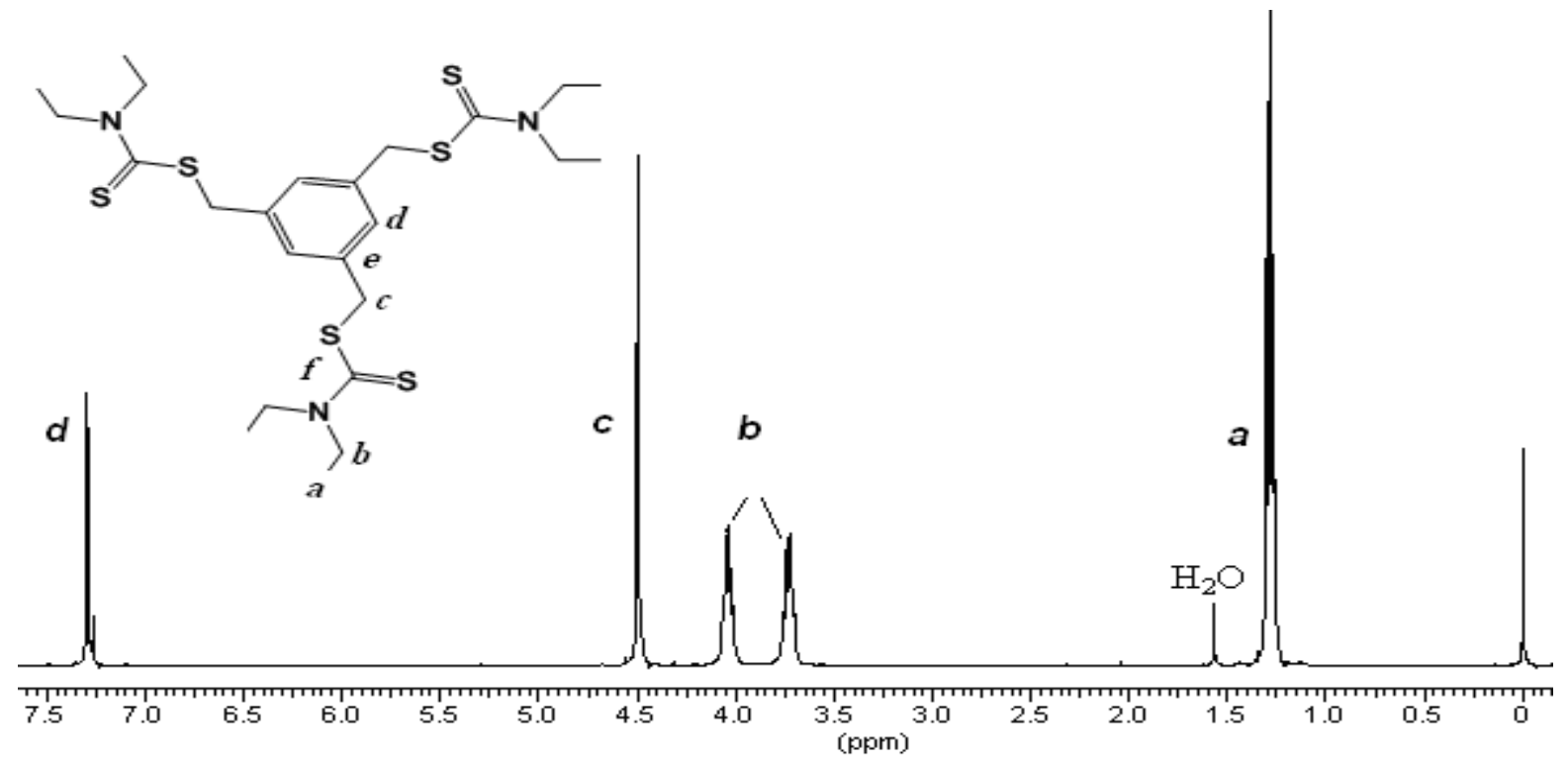

\subsection{Instruments}

The FT-IR spectrum was recorded in KBr pellets on a Digilab-FTS 2000 spectrometer. The UV-Vis absorption spectra were measured using an UV-Vis SPECORD 200 Analytik Jena spectrometer in $\mathrm{CHCl}_{3}$ as solvent. ${ }^{1} \mathrm{H}-\mathrm{NMR}$ and ${ }^{13} \mathrm{C}$-NMR spectra were recorded at room temperature on a Bruker Avance DRX 400 spectrometer (400 MHz and $100 \mathrm{MHz}$ ) as solutions in $\mathrm{CDCl}_{3}$ and chemical shifts are reported in ppm and referenced to TMS as internal standard. Molecular weights of the copolymers were determined by gel permeation chromatography method using THF as solvent and polystyrene as calibration standard. Microwave-assisted RAFT polymerization was performed in a single-mode microwave reactor (Discover Labmate System from CEM Corporation) equipped with a calibrated infrared temperature sensor (Maximum pressure $=20 \mathrm{bar}$, maximum power $=200 \mathrm{~W}$ ). All experiments were carried out in $10 \mathrm{~mL}$ standard Pyrex vials sealed with a special PTFE cap, in temperature-control mode and the temperature was controlled using the built-in calibrated IR sensor.

\subsection{Microwave RAFT Polymerizations of NVK}

An example of RAFT polymerization of NVK under microware heating conditions is as follows. A solution was prepared of NVK $(0.3865 \mathrm{~g}, 2 \mathrm{mmol})$, CTA [1,3,5-benzyl tri(diethyldithiocarbamate)] (5.6 mg, $0.01 \mathrm{mmol})$, AIBN (1.7 mg, $0.01 \mathrm{mmol})$ and 1,4-dioxane $(2.0 \mathrm{~mL})$. The aliquot was sealed in a $10 \mathrm{~mL}$ glass vial equipped with a magnetic stirrer bar. The resulting solution was purged with argon 
for $10 \mathrm{~min}$, the reaction vial was placed in the cavity of the microwave instrument, and the temperature was programmed to $70{ }^{\circ} \mathrm{C}$. After $25 \mathrm{~min}$, the polymerization was quenched by ceasing microwave irradiation, removing the vial from the instrument, rapidly cooling in an ice-water bath, and exposing the reaction solution to air. The polymer was isolated by precipitation in cold acetone, filtration, and drying under vacuum. PVK was purified by several precipitations from chloroform solution using acetone as non-solvent.

The theoretical number-average molecular weight is defined as follows (Equation 1):

$$
\left.\bar{M}_{n} \text { (theor. }\right)=\frac{[\text { Monomer }]_{0}}{[\mathrm{CTA}]_{0}} \times M_{\text {monomer }} \times \text { Conv. }+M_{\mathrm{CTA}}
$$

where: $M_{\mathrm{CTA}}$ and $M_{\text {monomer }}$ are the molecular weights of CTA (562) and of the monomer (193), and $[\text { Monomer }]_{0}$ and $[\mathrm{CTA}]_{0}$ are the initial concentrations of the monomer and CTA, respectively. Conversion was determined by gravimetry after the first precipitation in acetone.

\section{Results and Discussion}

To our best knowledge, the microwave-assisted RAFT polymerization of NVK has not been reported until now. However there is a short communication about the bulk polymerization reaction of NVK under microwave irradiation, in the presence of [60]fullerene, when initiation of polymerization takes place through the intermediate of the charge transfer complex formed between [60]fullerene and NVK [23].

The microwave-assisted reversible addition-fragmentation chain transfer polymerization of NVK was carried out in 1,4-dioxane as solvent using azobisisobutyronitrile (AIBN) as initiator at $70{ }^{\circ} \mathrm{C}$ and a dithiocarbamate derivative as trifunctional chain transfer agent (CTA) (Scheme 2). The control and monitoring of the temperature in the reaction vial was performed by a conventional infrared sensor and all polymerizations were carried out under the same experimental conditions. The temperature profile shows the set temperature is reached after 1-2 min.

Scheme 2. Microwave-assisted reversible addition-fragmentation chain transfer polymerization of $\mathrm{N}$-vinylcarbazole (NVK).
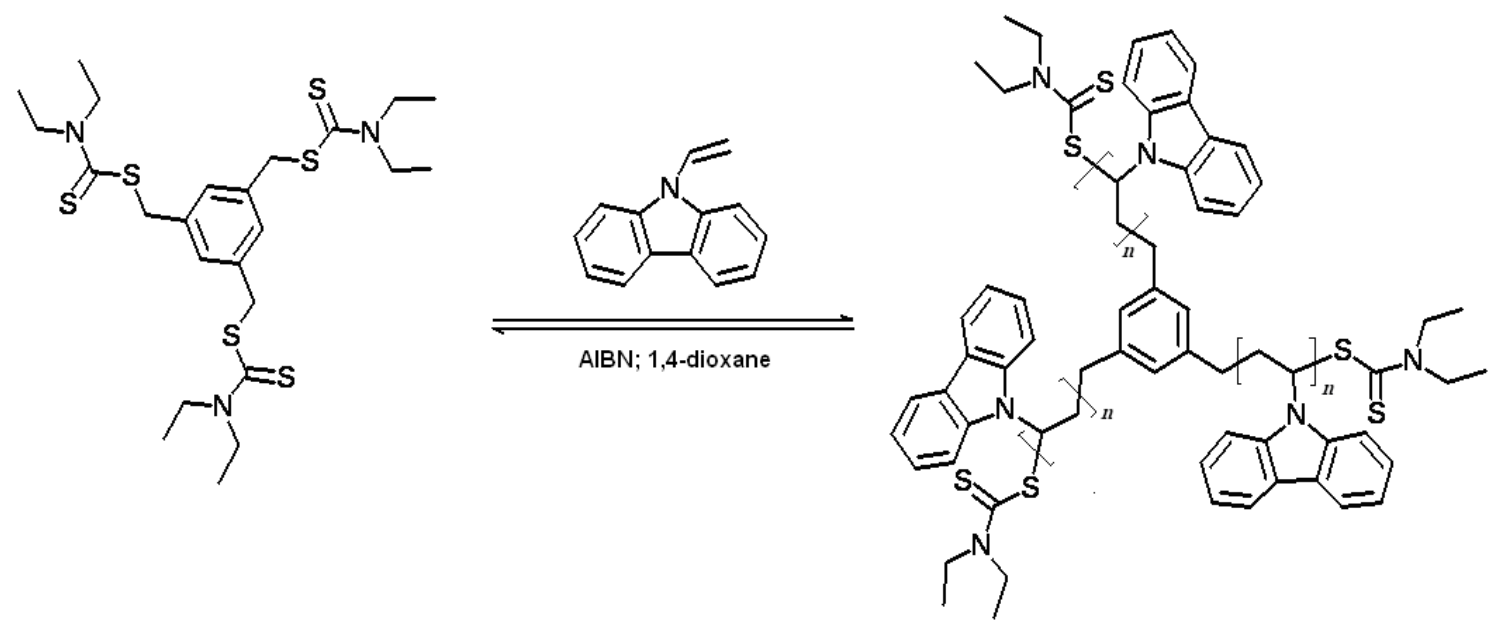
In the three-functionalized CTA, the leaving $\mathrm{R}$ group corresponds to $\mathrm{Ph}\left(\mathrm{CH}_{2}\right)_{3}$, which yields a radical species upon fragmentation, and $N, N$-diethyl as the $\mathrm{Z}$ group. We investigated the polymerization using different molar ratios between chain transfer agent-to-initiator, $[\mathrm{CTA}] /[\mathrm{AIBN}]=1,2,4$ and 5 , since the ratio can frequently be a critical factor in determining the overall success of a RAFT polymerization. Note that these CTA-to-AIBN ratios correspond to the dithiocarbamate moiety to AIBN ratios of 3,6,12 and 15 respectively, because of the multifunctional nature of the CTA having a triple concentration of dithiocarbonate groups. In all cases, the polymerization was conducted in 1,4-dioxane at $70{ }^{\circ} \mathrm{C}$, keeping the monomer-to-chain transfer agent ratio at a constant value of $[\mathrm{NVK}]_{0} /[\mathrm{CTA}]_{0}=200$. Under these conditions, the transparent solution was maintained without significant changes in the viscosity during the polymerization. Also, in other experiments, the RAFT polymerizations were carried out at different [Monomer $]_{0} /[\mathrm{CTA}]_{0}$ ratios: between $200 / 2-200 / 5$, or $100 / 1-500 / 1$, while the $[\mathrm{CTA}]_{0} /[\mathrm{AIBN}]_{0}$ molar ratio was held constant. The experimental results are given in Table 1.

Table 1. Synthesis of three-arm poly( $N$-vinylcarbazole) (PVK) by microwave-assisted reversible addition-fragmentation chain transfer (RAFT) polymerization of $N$-vinylcarbazole.

\begin{tabular}{|c|c|c|c|c|c|c|}
\hline Sample & $\begin{array}{l}\text { Time } \\
\text { (min) }\end{array}$ & {$[\mathrm{NVK}]_{0} /\left[[\mathrm{CTA}]_{0} /[\mathrm{AIBN}]_{0}\right.$} & $M_{\mathrm{n}}$ th. ${ }^{\mathrm{a}}$ & $\begin{array}{c}M_{\mathrm{n}}^{\mathrm{b}} \\
(\mathrm{g} / \mathrm{mol})\end{array}$ & $M_{\mathrm{w}} / M_{\mathrm{n}}{ }^{\mathbf{b}}$ & Conv. $(\%)^{\mathrm{C}}$ \\
\hline 1 & 5 & $400: 2: 1$ & 4468 & 7899 & 1.53 & 10.12 \\
\hline 2 & 10 & 400:2:1 & 9004 & 8120 & 1.42 & 21.87 \\
\hline 3 & 15 & $400: 2: 1$ & 13821 & 8436 & 1.382 & 34.35 \\
\hline 4 & 20 & $400: 2: 1$ & 17446 & 9789 & 1.26 & 43.74 \\
\hline 5 & 25 & 400:2:1 & 21664 & 11009 & 1.22 & 54.67 \\
\hline 6 & 30 & $400: 2: 1$ & 25887 & 14323 & 1.271 & 65.61 \\
\hline 7 & 35 & $400: 2: 1$ & 32310 & 16549 & 1.213 & 82.25 \\
\hline 8 & 25 & 200:2:1 & 5866 & 12517 & 1.382 & 27.48 \\
\hline 9 & 25 & 200:4:1 & 2308 & 9789 & 1.264 & 18.1 \\
\hline 10 & 25 & 200:5:1 & 2554 & 8436 & 1.229 & 25.8 \\
\hline 11 & 25 & 100:1:1 & 5866 & 12517 & 1.382 & 27.48 \\
\hline 12 & 25 & 300:1:1 & 12374 & 15509 & 1.680 & 20.4 \\
\hline 13 & 25 & 400:1:1 & 3125 & - & - & 3.32 \\
\hline 14 & 25 & 500:1:1 & 6333 & 16554 & 1.859 & 5.98 \\
\hline
\end{tabular}

${ }^{\mathrm{a}}$ Determined with Equation $1 ;{ }^{\mathrm{b}}$ Numer-average molecular weight $\left(M_{\mathrm{n}}\right)$ and molecular weight distribution $\left(M_{\mathrm{w}} / M_{\mathrm{n}}\right)$ were measured by gel chromatography (GPC) using polystyrene standards in THF; ${ }^{c}$ Conversion was approximated by gravimetry.

It can be observed that the number-average molecular weights of star PVK increase with the $[\text { Monomer }]_{0} /[\mathrm{CTA}]_{0}$ ratios. Consequently, control of the polymerization of NVK using three-functional CTA was confirmed by the formation of relatively narrow polydisperse products. The polydispersity indices $\left(M_{\mathrm{w}} / M_{\mathrm{n}}\right)$ for all samples prepared with three-functional CTA ranged between 1.22 and 1.85 (Table 1). However, dithiocarbamates used as CTAs provided PVK with broader polydispersities than xanthates [14-16]. A similar behavior was observed in RAFT polymerization of $N$-vinylindole with xanthates and dithiocarbamates as CTAs, explainable by the ratio between the leaving and re-initiation ability of the R-group [24]. 
Figures 2 and 3 show the variations of conversion and $\ln \left([\mathrm{M}]_{0} /[\mathrm{M}]_{\mathrm{t}}\right)$ vs. polymerization time, when $\mathrm{NVK}$ was polymerized at $[\mathrm{NVK}]_{0} /[\mathrm{CTA}]_{0} /[\mathrm{AIBN}]_{0}=400: 2: 1$.

Figure 2. Evolution conversion as a function of polymerization time (min). $[\mathrm{NVK}]_{0} /[\mathrm{CTA}]_{0} /[\mathrm{AIBN}]_{0}=400: 2: 1$ in 1,4 -dioxane at $70{ }^{\circ} \mathrm{C}$.

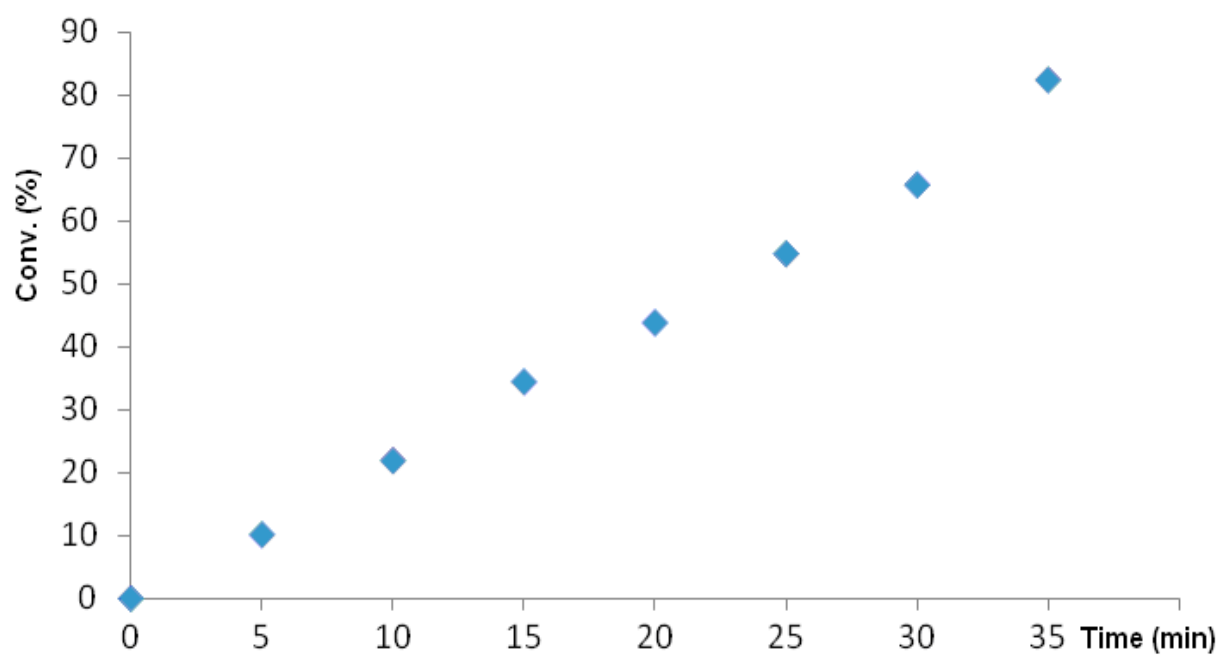

Figure 3. First-order kinetic plot for the polymerization of $N$-vinylcarbazole (NVK) with azobisisobutyronitrile (AIBN) in the presence of three-functional CTA at $[\mathrm{NVK}]_{0} /[\mathrm{CTA}]_{0} /[\mathrm{AIBN}]_{0}=400: 2: 1$ in 1,4 -dioxane at $70{ }^{\circ} \mathrm{C}$.

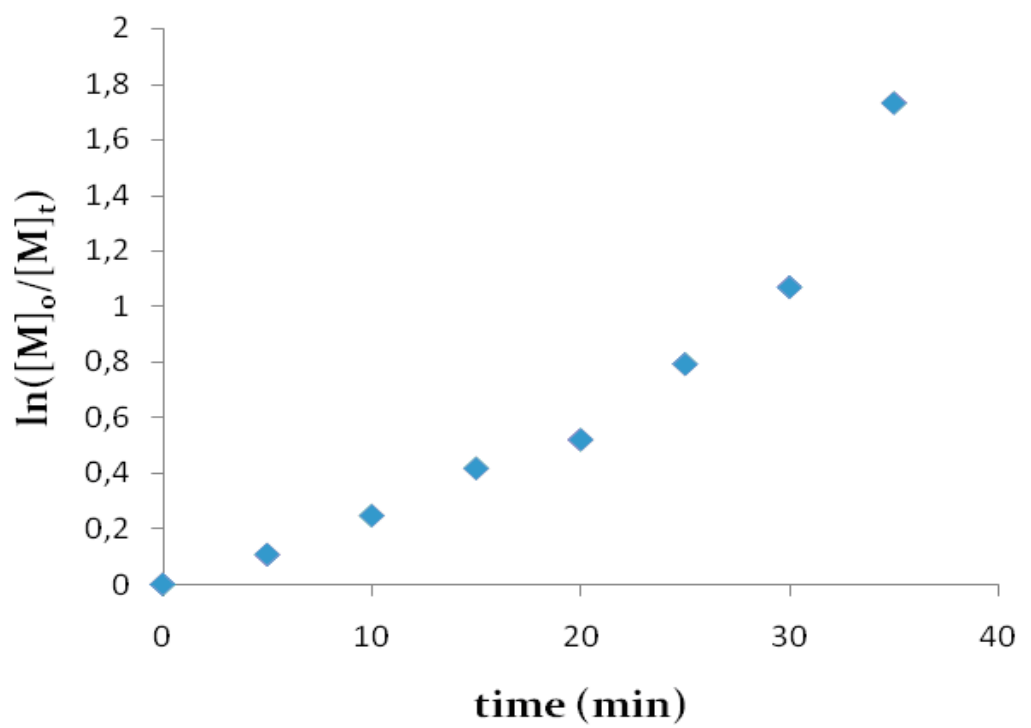

Nonlinear first-order kinetic plots are also observed, suggesting that radical concentrations are not constant. A number of possible explanations have been suggested, including slow fragmentation of the initiating leaving group radical, slow re-initiation by the expelled radical, increased stability of the intermediate radical (with and without intermediate radical termination), tendency of the expelled radical to add to the CTA rather than to monomer, and possible impurities in the CTA [13-17].

Figures 4 and 5 show the evolution of experimental molecular weight and polydispersity degree with monomer conversion. 
Figure 4. Evolution of experimental molecular weight with polymerization yield $[\mathrm{NVK}]_{0} /[\mathrm{CTA}]_{0} /[\mathrm{AIBN}]_{0}=400: 2: 1$ in 1,4 -dioxane at $70^{\circ} \mathrm{C}$.

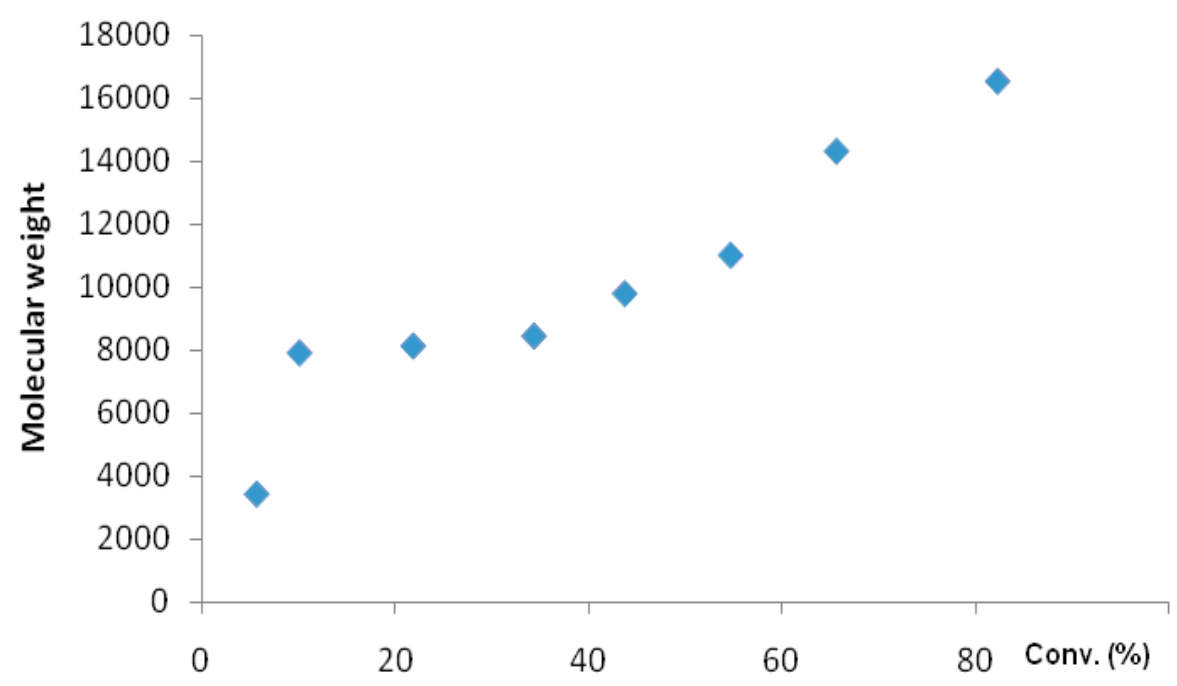

Figure 5. Evolution of polydispersity index with conversion: $[\mathrm{NVK}]_{0} /[\mathrm{CTA}]_{0} /[\mathrm{AIBN}]_{0}=400: 2: 1$ in 1,4-dioxane at $70^{\circ} \mathrm{C}$.

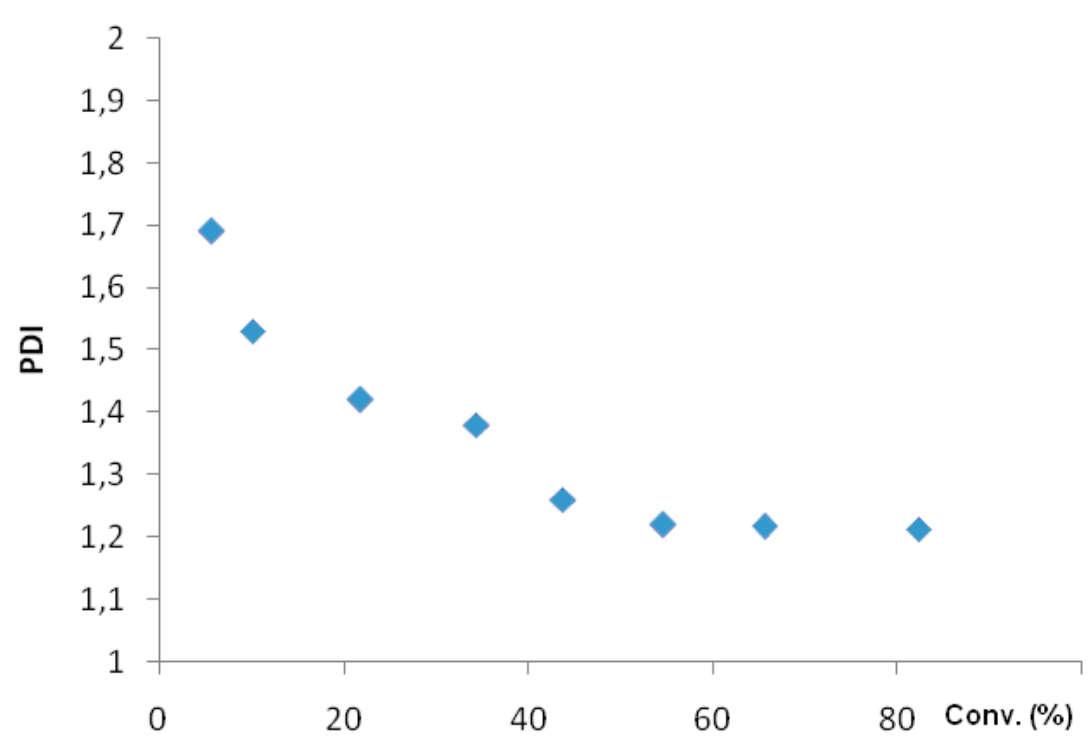

The quasi-linear increase of $M_{\mathrm{n}}$ with monomer conversion is normally evidenced for a controlled/living polymerization.

An analysis of the GPC curves (Figure 6) shows evolution of the PVK molecular weight distribution function of polymerization time (a) and monomer-to-chain transfer agent ratios (b), NVK being polymerized in the presence of the trifunctional RAFT agent. Each GPC trace of PVK obtained at short reaction times exhibits a monomodal distribution but at longer reaction times the distribution becomes bi- or even trimodal (Figure 6). It is evident that, as the polymerization proceeds, the main peak is accompanied by two minor shoulders assigned to the low molecular weight and high molecular weight polymers. All three distributions grow linearly with conversion, indicating that none of the distributions are due to the dead polymer. A multimodal distribution of the molecular weights was 
evidenced by Davis et al. in the RAFT polymerization of styrene using a hexa-functional CTA of a dithioester-type. The tri-modal molecular weight distribution can be explained as follows: the low molecular weight shoulder is due to linear non-star RAFT end-capped material, which is formed from fragments of the decomposing initiator, the main polymer peak is the three-arm ideal star material while the high molecular weight shoulder is due to polymer resulted from star-star coupling caused by radical-radical termination. However, more experimental studies have to be conducted to confirm these assumptions.

Figure 6. Molecular weight distributions for PVK star with: (a) polymerization time (Table 1, entry 1, 3 and 7), and (b) using different monomer-to-chain transfer ratios; polymerization time $=25 \mathrm{~min}$. (Table 1 , entry 5, 8, 9 and 10).
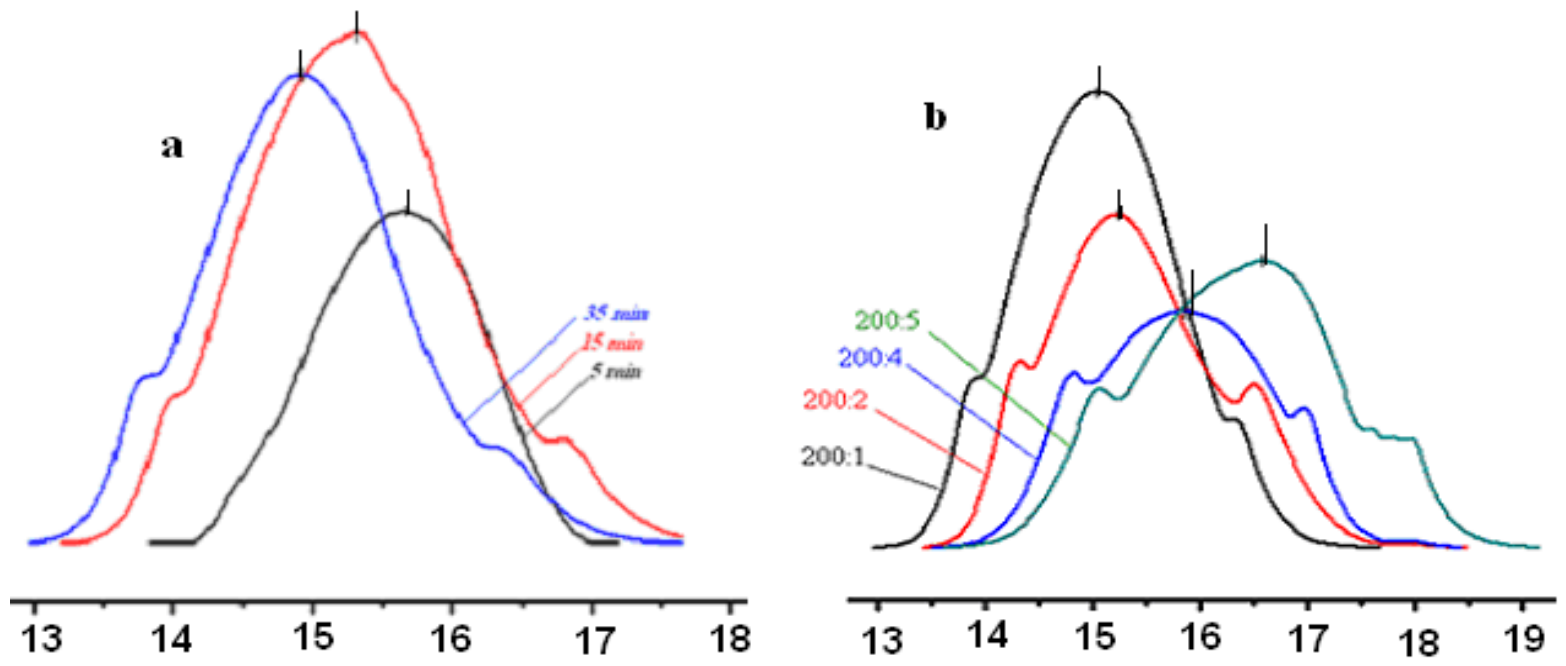

Elution time (min)

In most cases, the molecular weights of the poly(NVK) star, measured by GPC in THF, were lower than the theoretical values calculated from the monomer/CTA molar ratio and conversion of the monomer. The molecular weights obtained by conventional GPC using polystyrene calibration are just the apparent ones due to the compact nature of the branched macromolecules and the lack of suitable standards. Moreover, the conversions were determined by a gravimetry method and some errors are introduced. It is clear that after the first precipitation in acetone, low oligomers and unreacted monomer are removed and molecular weights are increased artificially. At the same time, conversion is lower than the real value due to the loss of some oligomers.

Figure 7 shows a typical ${ }^{1} \mathrm{H}$ NMR spectrum of PVK synthesized by the reversible addition-fragmentation chain transfer (RAFT) method with a microwave reactor. The ${ }^{1} \mathrm{H}-\mathrm{NMR}$ spectra of RAFT-synthesized PVK (Figure 7) shows aromatic protons over a wide range (6-8.5 ppm) due to the hindered rotation and shielding effects, associated with the bulkiness of carbazole groups, while aliphatic protons are observed between $0.8-2.5 \mathrm{ppm}$. This spectrum is reminiscent of the classical spectrum of cationic and radical-synthesized poly( $N$-vinylcarbazole), where also signals are observed between 5-8.5 ppm and are assigned to aromatic protons shifted at high field due to shielding [25]. 
Unfortunately, attempts to evaluate conversion and the structure of the end groups by NMR were unsuccessful because the peaks assigned to different protons are broad and overlapped the others.

Figure 7. ${ }^{1} \mathrm{H}-\mathrm{NMR}$ spectra $\left(\mathrm{CDCl}_{3}\right)$ of the poly(NVK) (Sample 6 from Table 1). * are assigned to methanol traces.

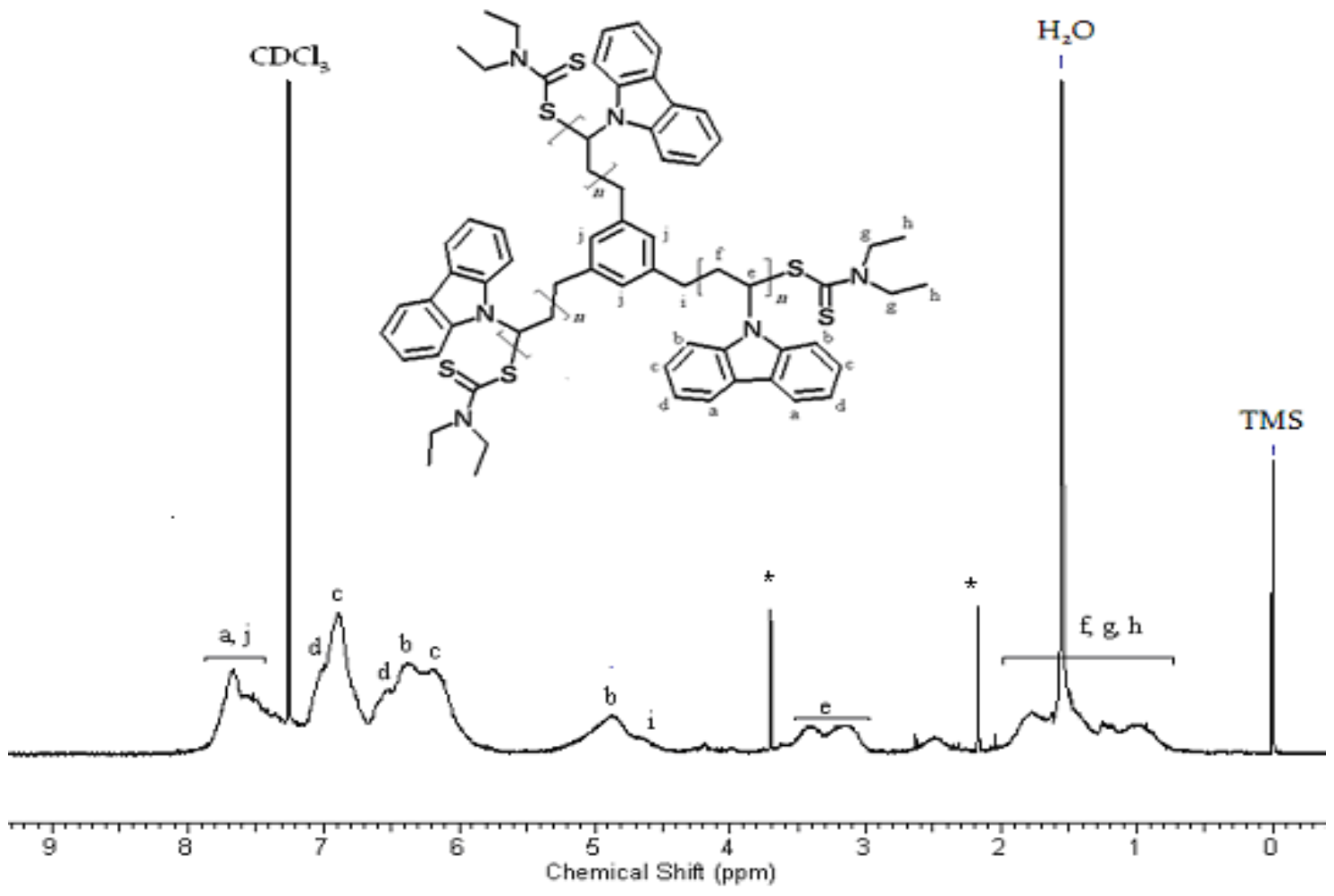

\section{Conclusions}

Poly( $N$-vinylcarbazole) with star architecture was synthesized via a reversible addition-fragmentation chain transfer polymerization using a three-functional chain transfer agent of a dithiocarbamate-type, 1,3,5-benzyl tri(diethyldithiocarbamate). The RAFT polymerization method can be applied to the polymerization of vinyl monomers with bulky and strong electronodonor groups to obtain polymers having a moderate control of molecular weights and polydispersity indices, suitable for use in block copolymer synthesis and for optoelectronic studies.

\section{Acknowledgments}

Authors thank to the Romanian National Authority for Scientific Research (UEFISCDI) for financial support (Grant PN-II-ID-PCE-2011-3-0274, Contract 148/2011).

\section{References}

1. Grazulevicius, J.V.; Strohriegl, P.; Pielichowski, J.; Pielichowski, K. Carbazole-containing polymers: synthesis, properties and applications. Prog. Polym. Sci. 2003, 28, 1297-1353.

2. Simionescu, C.I.; Grigoras, M. Macromolecular charge transfer complexes. Prog. Polym. Sci. 1991, 16, 907-976. 
3. Schaffert, R.M. A new high-sensitivity organic photoconductor for electrophotography. IBM $J$. Res. Dev. 1971, 15, 75-89.

4. Georges, M.K.; Veregin, R.P.N.; Kazmaier, P.M.; Hamer,G.K. Narrow molecular weight resins by a free-radical polymerization process. Macromolecules 1993, 26, 2987-2988.

5. Hawker, C.J. Molecular weight control by a "living" free-radical polymerization process. J. Am. Chem. Soc. 1994, 116, 11185-11186.

6. Kato, M.; Kamigaito, M.; Sawamoto, M.; Higashimura, T. Polymerization of methyl methacrylate with the carbon tetrachloride/dichlorotris-(triphenylphosphine)ruthenium (II)/methylaluminium bis(2,6-di-tert-butylphenoxide) initiating system: possibility of living radical polymerization. Macromolecules 1995, 28, 1721-1723.

7. Wang, J.S.; Matyjaszewski, K. Controlled/living radical polymerization. Atom Transfer Polymerization in the presence of transition metal complexes. J. Am. Chem. Soc. 1995, 117, 5614-5615.

8. Matyjaszewski, K.; Xia, J.H. Atom transfer radical polymerization. Chem. Rev. 2001, 101, 2921-2990.

9. Percec, V.; Grigoras, C. Arenesulfonyl Iodides: The third universal class of functional initiators for the metal-catalyzed living radical polymerization of methacrylates and styrenes. J. Polym. Sci. Part A: Polym. Chem. 2005, 43, 3920-3931.

10. Chiefari, J; Chong, Y.K.; Ercole, F.; Krstina, J.; Jeffery, J.; Le, T.P.T.; Mayadunne, R.T.A.; Meijs, G.F.; Moad, C.L.; Moad, G.; et al. Living free-radical polymerization by reversible addition-fragmentation chain transfer: the RAFT process. Macromolecules 1998, 31, 5559-5562.

11. Mayadunne, R.T.A.; Rizzardo, E.; Chiefari, J.; Chong, Y.K.; Moad, G.; Thang, S.H. Living radical polymerization with reversible addition-fragmentation chain transfer (RAFT polymerization) using dithiocarbamates as chain transfer agents. Macromolecules 1999, 32, 6977-6980.

12. Chong, Y.K.; Le, T.P.T.; Moad, G.; Rizzardo, E.; Thang, S.H. A more versatile route to block copolymers and other polymers of comples architecture by living radical polymerization: The RAFT process. Macromolecules 1999, 32, 2071-2074.

13. Moad, G.; Chiefari, J.; Chong, Y.K.; Krstina, J.; Mayadunne, R.T.A.; Postma, A.; Rizzardo, E.; Thang, S.H. Living free radical polymerization with reversible addition-fragmentation chain transfer (the life of RAFT). Polym. Int. 2000, 49, 993-1001.

14. Mori, H.; Ookuma, H.; Endo, T. Poly( $N$-vinylcarbazole) star polymers and amphiphilic star block copolymers by xanthate-mediated controlled radical polymerization. Macromolecules 2008, 41, 6925-6934.

15. Mori, H.; Ookuma, H.; Nakano, S.; Endo, T. Xanthate-mediated controlled radical polymerization of $N$-vinylcarbazole. Macromol. Chem. Phys. 2006, 207, 1005-1017.

16. Hu, N.; Ji, W.X.; Tong, Y.Y.; Li, Z.C.; Chen, E.Q. Synthesis of diblock copolymers containing poly $(N$-vinylcarbazole) by reversible-addition-fragmentation chain transfer polymerization. J. Polym. Sci. Part A: Polym. Chem. 2010, 48, 4621-4626.

17. Kappe, C.O.; Dallinger, D.; Murphree S.S. Practical Microwave Synthesis for Organic Chemists: Strategies, Instruments and Protocols, 1st ed.; Wiley-VCH Verlag GmbH \& Co.KgaA, Weinheim: Weinheim, Germany, 2009. 
18. Herrero, M.A.; Kremsner, J.M.; Kappe, C.O. Nonthermal microwave effects revisited: On the importance of internal temperature monitoring and agitation in microwave chemistry. J. Org. Chem. 2008, 73, 36-47.

19. Roy, D.; Ullah, A.; Sumerlin, B.S. Rapid block copolymer synthesis by microwave-assisted RAFT polymerization. Macromolecules 2009, 42, 7701-7708.

20. Mayadunne, R.T.A.; Jeffery, J.; Moad, G.; Rizzardo, E. Living free radical polymerization with reversible addition fragmentation chain transfer (RAFT polymerization): Approaches to star polymers. Macromolecules 2003, 36, 1505-1513.

21. Bernard, J.; Favier, A.; Zhang, L.; Nilasaroya, A.; Davis, T.P.; Barner-Kowollik, C.; Stenzel, M.H. Poly(vinyl ester) star polymers via xanthate-mediated living radical polymerization: From poly(vinyl alcohol) to glycopolymers stars. Macromolecules 2005, 38, 5475-5484.

22. Stenzel-Rosenbaum, M.; Davis, T.P.; Chen, V.; Fane, A.G. Star-polymer synthesis via radical reversible addition-fragmentation chain transfer polymerization. J. Polym. Sci.: Part A: Polym. Chem. 2001, 39, 2777-2783.

23. Chen, Y.; Wang, J.; Zhang, D.; Cai, R.; Yu, H.; Su, C.; Huang, Z.E. [60] Fullerene-initiated bulk polymerization of $\mathrm{N}$-vinylcarbazole under the microwave irradiation. Polymer 2000, 41, 7877-7880.

24. Maki, Y.; Mori, H.; Endo, T. Xanthate-mediated controlled radical polymerization of $N$-vinylindole derivatives. Macromolecules 2007, 40, 6119-6130.

25. Williams, D.J. Nuclear magnetic resonance studies of poly( $N$-vinylcarbazole). Macromolecules 1970, 3, 602-605.

(C) 2012 by the authors; licensee MDPI, Basel, Switzerland. This article is an open access article distributed under the terms and conditions of the Creative Commons Attribution license (http://creativecommons.org/licenses/by/3.0/). 